\title{
A BROWSER EXTENSION FOR PROVIDING VISUALLY IMPAIRED USERS ACCESS TO THE CONTENT OF BAR CHARTS ON THE WEB
}

\author{
Stephanie Elzer, Edward Schwartz \\ Department of Computer Science, Millersville University, POB 1002, Millersville, PA 17551, USA \\ elzer@cs.millersville.edu,ejschwartz@cs.millersville.edu \\ Sandra Carberry, Daniel Chester, Seniz Demir, Peng Wu \\ Department of Computer \& Information Sciences, University of Delaware, 103 Smith Hall, Newark, DE 19716, USA \\ carberry@cis.udel.edu,chester@cis.udel.edu,demir@cis.udel.edu,pwu@cis.udel.edu
}

Keywords: Accessibility, assistive technology, plan recognition, Bayesian networks, graph understanding

Abstract: $\quad$ This paper presents the SIGHT (Summarizing Information GrapHics Textually) system that enables visually impaired users to gain access to information graphics that appear on a web page. The user interface is implemented as a browser extension that is launched by a keystroke combination. The output of SIGHT is a textual summary of the graphic, the core content of which is the hypothesized intended message of the graphic designer. The textual summary of the graphic is then conveyed to the user by screen reading software (such as JAWS). This approach has the benefits of 1) not requiring any action on the part of the web page developer, and 2) providing the user with the message and knowledge that one would gain from viewing the graphic rather than requiring the user to form a mental map of the graphic.

\section{INTRODUCTION}

Information graphics (non-pictorial graphics, such as bar charts, line graphs and pie charts) are an important component of many documents. Unfortunately, these visual presentations present serious information access challenges for visually impaired users. Documents on the Web can be accessed by visually impaired users through screen reading software that reads the information on a computer screen using synthesized speech. If the developer of the web page has supplied alternative text (or "alt text") for graphics in the html, most screen readers will read this text to the user. However, the vast majority of web pages are developed without broad accessibility in mind, and alt text is not supplied, thus making the content of the document's graphics inaccessible to a visually impaired user.

It has been our observation that the majority of information graphics that appear in formal reports, newspapers, and magazines are intended to convey a message or communicative intention. We conducted a corpus study (Carberry et al., 2006) whose primary goal was to determine the extent to which the message conveyed by an information graphic in a multimodal document is also conveyed by the document's text. We analyzed 100 randomly selected graphics from our collected corpus of information graphics, along with the articles in which they appeared. The selected articles were taken from magazines (such as Newsweek, Business Week, Fortune, and Time) and local and national newspapers. In 39\% of the instances, the text was judged to fully or mostly convey the message of the information graphic. However, in $26 \%$ of the instances, the text conveyed only a little of the graphic's message. Most surprising was the observation that in $35 \%$ of the instances in our analyzed corpus, the text failed to convey any of the message. These findings make it crucial that mechanisms be developed for providing visually impaired individuals with alternative access to the content of information graphics.

A number of projects have attempted to make images accessible to visually impaired viewers by reproducing the image in an alternative medium, such as sound (Meijer, 1992; Alty and Rigas, 1998), touch (Ina, 1996) or a combination of the two (Ramloll et al., 2000; Yu et al., 2002; Kennel, 1996). One particularly interesting project is that of Yu and Brewster (Yu et al., 2002). In this project, they investigate the development and usefulness of web-based multimodal graphs which use haptic devices and audi- 
tory output to communicate the contents of the graphs to the users. The web pages containing the graphics must be properly formatted with the coordination of embedded haptic and audio features controlled by Cascading Style Sheets (Yu et al., 2002). Although the evaluation of their system does demonstrate the usefulness of the approach when compared to traditional tactile diagrams, they note that the process of preparing the graphics is laborious (Yu et al., 2002).

Aside from the use of sound and touch, there is some research by Kurze (Kurze, 1995) that generates text in a presentation tool used to convey the content of a graphic. In this system, a verbal description of the diagram's properties, such as the style of the diagram, the number of data sets, the labels of axes and the ranges of axes, is output through a text-to-speech device (Kurze, 1995).

However, all of these approaches require the user to build a "mental map" of the diagram - a task that is very difficult for the congenitally blind because they have no personal knowledge regarding the appearance of information graphics (Kennel, 1996). In addition, many of the other systems require 1) special equipment or 2) preparation work (model creation) to be done by a sighted individual. Consequently, existing systems have not been successful in solving the accessibility issue for visually impaired individuals. Thus it is imperative that novel approaches be investigated.

Rather than providing alternative access to what the graphic looks like or a listing of all of the data points contained in the graphic, our SIGHT system provides the user with the message and knowledge that one would gain from viewing the graphic. We hypothesize that the core message of an information graphic (the primary overall message that the graphic conveys) can serve as the basis for an effective textual summary of the graphic. For example, given the graphic in Figure 1, rather than simply presenting the heights of the bars and their values, SIGHT infers that the graphic's intended message is that there is an increase in Jan '99 in the dollar value of 6-month growth in consumer revolving credit in contrast with the decreasing trend from July '97 to July '98 and conveys this message to the user via speech. We eventually envision SIGHT as an interactive natural language system that will provide a richer textual summary, with the inferred message as the core content, and that can respond to follow-up questions from the user or requests for justification of the system's inferred message.

The remainder of the paper presents our implemented SIGHT system. First we present the browser extension that we have constructed to enable visually

\footnotetext{
${ }^{1}$ Graphic from BusinessWeek, April 5, 1999 issue.
}

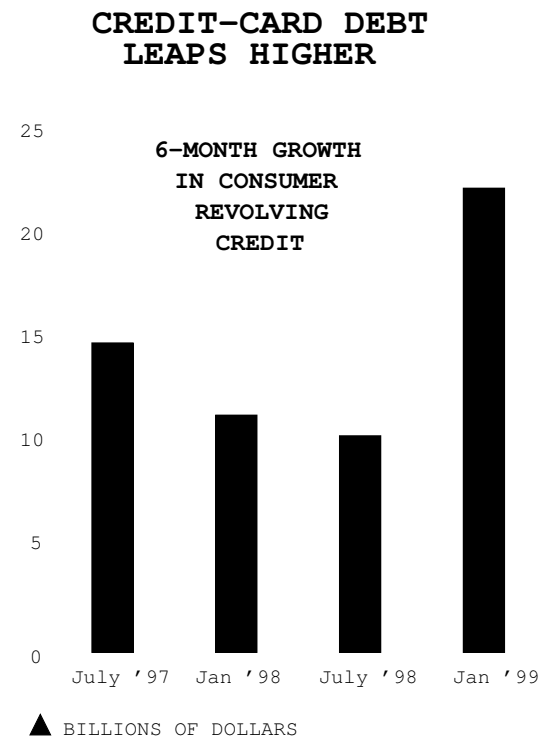

Figure 1: Graphic with a Contrast Pt with Trend Message ${ }^{1}$

impaired users to gain access to the information provided by information graphics that appear on a web page. Next we outline how images are translated into an XML representation of the graphic, and then we present our overall methodology for inferring the intended message of an information graphic. Note that all of the examples presented in this paper have been successfully run on the SIGHT system. We then conclude with a discussion of the future directions of our work.

\section{THE SIGHT SYSTEM}

The architecture of our SIGHT system is shown in Figure 2. The following subsections discuss the various components with particular emphasis on the browser extension which allows visually impaired users to access textual summaries of information graphics.

\subsection{The Browser Extension}

Because our target users are visually impaired, our user interface cannot rely on any of the usual visual mechanisms. Instead, the users must be able to launch our application from the keyboard. We have implemented our browser extension specifically for JAWS 7.10 (the most recently released version of JAWS) and Internet Explorer (preferably version 6.0 or later). JAWS, produced by Freedom Scientific, is the most widely used screen reading software for Windows, 


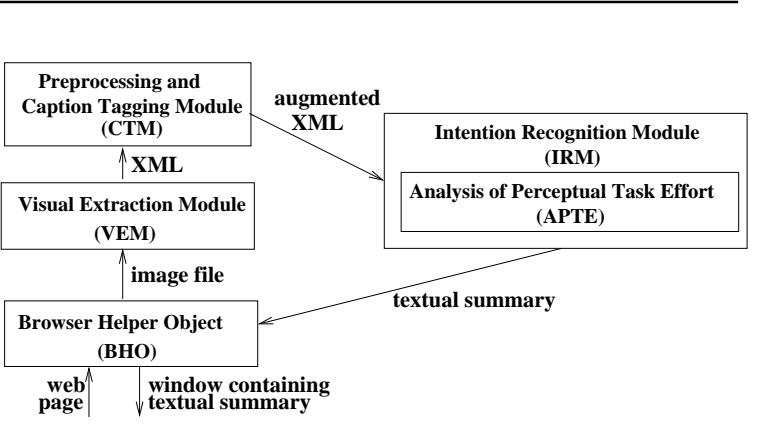

Figure 2: SIGHT System Architecture

and JAWS users are encouraged to use Microsoft's Internet Explorer for web browsing, so this pair of applications should be ideal for a large portion of our target users. However, the concepts applied here are extensible to other implementation platforms, as explained in Section 2.2.3.

When navigating a web page, JAWS users have many options. When the web page is initially opened, JAWS begins reading the content of the web page, from top to bottom. The actual content that JAWS reads is highly configurable by the user, but typically includes any text on the page, the screen text pertaining to links and buttons, and the alternative text associated with graphics. So that we do not conflict with the existing navigation commands in JAWS, we chose $C O N T R O L+Z$ as the key combination for launching our interface. If the user comes across a bar chart during their navigation of a web page, they can hit CONTROL $+Z$ to launch our application and receive a textual summary of the information conveyed by the bar chart. For example, if the user encountered the graphic shown in Figure 3, they could hit CONTROL $+\mathrm{Z}$ and a new window containing the summary of the graphic would appear. For this particular graphic, SIGHT produces the summary

This bar chart titled 'The notebook spiral' shows that the dollar value of average laptop prices fell from 2000 to 2003 and then falls more slowly until 2007.

However, this type of interaction requires a very tight coupling between our application and the web browser, because our application needs to be able to determine which graphic is currently in focus within the web browser. We achieved the proper level of integration by implementing our user interface as a Browser Helper Object for Internet Explorer.

Browser Helper Objects are special add-on components that enable the customization of Internet Ex-

\footnotetext{
${ }^{2}$ Graphic from BusinessWeek, September 5, 2005.
}

THE NOTEBOOK SPIRAL

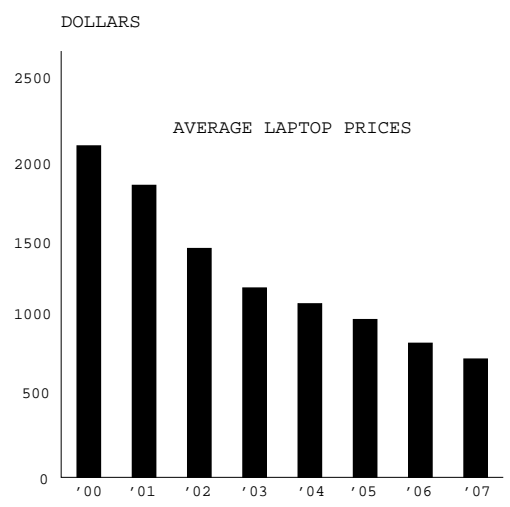

Figure 3: Graphic with a Changing Trend Message ${ }^{2}$

plorer (version 4.0 or later). BHOs are tied to the main window of the browser and are created as soon as a new browser window is created. BHOs are implemented as in-process Component Object Model $(\mathrm{COM})$ components, and they run in the same process space as the browser; this means that they can perform virtually any action on the available windows and modules. Our BHO hooks onto Internet Explorer's message loop and captures all of the keyboard events within the browser, looking for the CONTROL $+\mathrm{Z}$ combination. Upon detecting the CONTROL $+Z$ combination, the BHO queries the Document object of the Internet Explorer instance to determine which object is currently in focus within the browser. If the object in focus appears to be a graphic containing a bar chart, our system then attempts to infer the intended message of the bar chart. The resultant textual summary is presented to the user in a new window. The text in that new window is then read to the user by JAWS, and the user can subsequently close the window using standard JAWS/Windows keystrokes (typically ALT+F4).

\subsection{Implementation Issues}

The procedure described above works well as long as 1) the object currently in focus in the browser is a bar chart, and 2) the object in focus in the JAWS interface is the same as the object in focus according to the browser. This section addresses these issues.

\subsubsection{Identifying Bar Charts}

Since our BHO is operating within Internet Explorer, and web pages contain many graphical elements that are not bar charts, it is entirely possible that the user 
may attempt to launch our system to process a graphic that is not a bar chart. Therefore, when the CONTROL $+\mathrm{Z}$ keystroke is detected, the BHO runs a brief image processing algorithm to determine whether the selected graphic has particular attributes that identify it as a possible bar chart, such as whether the graphic has 20 or fewer gray levels, and whether the graphic contains at least two rectangles with a common beginning row or column. If the graphic does not appear to be a bar chart, the message "The selected graphic does not appear to be a bar chart," is read to the user by JAWS.

\subsubsection{Focusing on Bar Charts}

When browsing a web page in Internet Explorer, JAWS users have the option of "tabbing" through the content of the page. By repeatedly hitting the tab button, JAWS will traverse the elements on the page according to the browser's default tab order for that page or the tab order specified in the html code. When the user is traversing a web page in JAWS using the tab keys, the focus in Internet Explorer is updated along with JAWS' focus. For graphics that are in the tab order, JAWS will read the alt text (if any) associated with the graphic when the user traverses to the graphic. If the user hits CONTROL $+Z$ when JAWS reaches the graphic, our $\mathrm{BHO}$ will be activated.

However, many graphics are not included in the tab order of the page, since there is typically not an action associated with them (such as a link to follow or a text box to fill in). To address this issue, when a web page is opened in Internet Explorer, our BHO performs a scan of all of the graphics on that page, performing the bar chart detection logic described previously. If an image appears to be a bar chart, our $\mathrm{BHO}$ will insert that graphic into the tab order of the page, and will append "This graphic appears to be a bar chart" to the alt text (if any). This pre-processing of the web page ensures that the user will not accidentally overlook bar charts that could be processed by our system while traversing the page.

When a web page is opened in Internet Explorer while JAWS is running, JAWS automatically begins reading the content of the page from top to bottom (this is sometimes called "say all" mode). It is generally more convenient for JAWS users to utilize the "say all" mode rather than tabbing through the content of the page. If the BHO's pre-processing has detected a potential bar chart, the user will be alerted to its presence by JAWS reading the alt text, "This graphic appears to be a bar chart," that was inserted into the document by our BHO. Unfortunately, while in the "say all" mode, the focus in JAWS is not reflected in Internet Explorer. This obviously poses a problem since our BHO relies on the Document object in Internet Explorer to identify the graphic in which the user is interested. Fortunately, the latest release of JAWS allows the user to set the application focus to the location of the JAWS virtual cursor by entering CONTROL+INSERT+DELETE. After entering this while JAWS' virtual cursor has the graphic containing a bar chart in focus, the user may then enter CONTROL $+Z$ to hear the summary of the bar chart.

\subsubsection{Extensibility of the Browser Extension}

While the current version of the user interface has been designed specifically with JAWS and Internet Explorer in mind, we expect similar solutions to work for other applications. For example, extensions similar to BHOs can be developed for Mozilla's Firefox browser using the Cross Platform Component Object Model (XPCOM). Regarding the use of screen readers other than JAWS, our BHO in Internet Explorer will work with any screen reader; it is simply a matter of investigating how the focus of Internet Explorer and the screen reading software interact and of ensuring that the keystroke combination does not conflict with existing screen reader functionality. For visually impaired users who primarily use a screen magnifier (such as ZoomText), rather than a screen reader, the text produced by our BHO can be handled in the same manner as text in any other application.

\subsection{Processing the Image}

Once the browser component of the SIGHT system has detected that the user would like to access a particular graphic, it sends the image to the Visual Extraction Module. VEM is responsible for analyzing the graphic's image file and producing an XML representation containing information about the components of the information graphic including the graphic type (bar chart, pie chart, etc.) and the textual pieces of the graphic (such as its caption). For a bar chart, the representation includes the number of bars in the graph, the labels of the axes, and information for each bar such as the label, the height of the bar, the color of the bar, and so forth (Chester and Elzer, 2005). This module currently handles only electronic images produced with a given set of fonts and no overlapping characters. In addition, the VEM currently assumes standard placement of labels and axis headings. Work is underway to remove these restrictions. But even with these restrictions removed, the VEM can assume that it is dealing with a simple bar chart, and thus the problem of recognizing the entities in a graphic is much more constrained than typical computer vision problems. 
The XML representation is then passed to the Preprocessing and Caption Tagging Module (CTM). The preprocessing augments the $\mathrm{xml}$ with salience information such as a bar that is colored differently from other bars in the graphic or a bar that has an annotation when the other bars do not. The caption tagging extracts information from the caption (discussed later) and then passes the augmented XML representation to the intention recognition module (IRM), which is described in the next section.

\subsection{IRM: A Bayesian Inference System}

The IRM is responsible for recognizing the intended message of the information graphic, which we hypothesize can serve as the basis for an effective summary of the graphic. While the browser extension will work for any type of information graphic, the scope of the work currently implemented for the image processing and IRM components is limited to the processing of simple bar charts. By simple bar charts, we mean bar charts that display the values of a single independent attribute and the corresponding values for a single dependent attribute. Although our system currently handles only simple bar charts, we believe that our methodology is broadly applicable and extensible to other types of graphics.

\subsubsection{Communicative Signals}

We view information graphics that appear in popular media as a form of language with a communicative intention. The IRM reasons about the communicative signals present in a graphic in order to identify its intended message. We have identified three kinds of communicative signals that appear in simple bar charts.

Our first communicative signal is the relative effort required for different perceptual and cognitive tasks. ${ }^{3}$ Here we are extending a hypothesis of the AutoBrief group (Kerpedjiev and Roth, 2000). The AutoBrief project was concerned with generating information graphics, and they hypothesized that a graphic designer chooses a design that best facilitates the perceptual and cognitive tasks that a viewer will need to perform on the graphic. Thus given a graphic, our hypothesis is that the relative difficulty of different perceptual tasks serves as a signal about which tasks the

\footnotetext{
${ }^{3}$ By perceptual tasks(Kerpedjiev and Roth, 2000) we mean tasks that are performed by viewing the graphic, such as comparing the heights of two bars; by cognitive tasks, we mean tasks that require a mental computation such as interpolating between two labelled values on the dependent axis in order to determine the value represented by a bar whose top is not aligned with a labelled value.
}

viewer was expected to perform in deciphering the graphic's message. This correlates with Larkin and Simon's (Larkin and Simon, 1987) observation that graphics that are informationally equivalent are not necessarily computationally equivalent - for example, if a set of bars are arranged in order of increasing height, then it will be much easier to identify the rank of an individual bar than if the bars were arranged in alphabetical order of their label.

To rank tasks in terms of effort, we constructed a set of effort estimation rules. Each rule represents a task that can be performed on a graphic and is comprised of a set of condition-computation pairs: each condition captures possible features of the graphic (such as whether the top of a bar is aligned with a labelled tick mark on the dependent axis), and the computation captures the effort required for the task given that set of graphic features. The effort computations are based on work by cognitive psychologists, and our effort estimation rules have been validated via a set of eye-tracking experiments (Elzer et al., 2006).

A second communicative signal is the salience of entities in the graphic. Our system recognizes a number of ways in which an entity in a bar chart becomes salient. These include coloring or annotating a bar differently from the other bars in a bar chart (as is the case for the bar labelled CBS in Figure 4), since these design strategies draw attention to the bar. It also includes mentioning a bar's label in the caption, since this also draws attention to the bar. The preprocessor and caption tagging module are responsible for identifying salient entities in the graphic. The preprocessor analyzes the XML representation of the graphic and augments it to indicate entities that are salient due to graphic design decisions. The Caption Tagging Module uses a part-of-speech tagger to identify nouns in the caption, and then it augments the XML representation to indicate any bars that match a noun in the caption.

A third communicative signal is the presence of certain verbs and adjectives in a caption. In (Elzer et al., 2005a) we present a corpus study showing that (1) captions are often very general or uninformative, and (2) even when captions convey something about the graphic's intended message, the caption is often ill-formed or requires extensive analogical reasoning. Thus we have chosen to perform a shallow analysis of captions that extracts communicative signals but does not attempt to understand the caption. For example, the verb lag in the caption "American Express' total billings still lag” suggests a message about an entity's rank with respect to some measure. Similarly, we found that nouns derived from verbs, such as rise in the caption "Cable on the Rise", and adjectives 
also suggest the general category of message. Using WordNet and a thesaurus, we identified verbs and adjectives that were similar in meaning and might signal one or more categories of message and organized them into verb classes. The Caption Tagging Module uses a part-of-speech tagger and a stemmer to analyze captions and extract nouns, adjectives, and the root form of verbs, adjectives, and nouns derived from verbs, and further augments the XML representation of the graphic to indicate the presence of one of our identified verb or adjective classes in the caption.

\subsubsection{Reasoning about the Graphic's Intention}

Having developed a means of extracting the evidence provided by the communicative signals in a graphic, we need to use this evidence in reasoning about the intended message of an information graphic. For this purpose, we dynamically construct a Bayesian network for each new information graphic. The top level of the network captures the various categories of messages that can be conveyed by a bar chart, such as conveying a change in trend (Change-Trend), conveying the rank of an entity in a bar chart (Get-Rank), comparing two entities (Relative-Difference), etc. Below each category of message are nodes that capture the different possible instantiations of that message category. For example, if a graphic has five bars as in Figure 4 , then the children of the Get-Rank node would be Get-Rank(BAR1,LABEL1), ..., Get-Rank(BAR5, LABEL5).

We use plan operators to specify how a graphic designer's communicative goal can be achieved via the viewer performing certain perceptual and cognitive tasks. For example, consider the goal of the viewer getting the rank of a bar, given that the bar is salient, as in Figure 4. The operator for achieving this goal decomposes the goal into three subgoals: perceiving whether the bars are sorted in order of height, perceiving (ie., finding) the label associated with the bar, and perceiving the rank of that bar with respect to bar height. Each subgoal in an operator is either a primitive with an associated effort rule or has an operator that decomposes it into a set of simpler subgoals. The operators determine the structure of our Bayesian network, in that subgoals in an operator become children of their goal node in the Bayesian network. For example, Figure 5 displays the piece of the Bayesian network produced by the Get-Rank operator. The entire network is built dynamically for a new graphic.

\footnotetext{
${ }^{4}$ Graphic from BusinessWeek, April 5, 1999. Note that in its original form, the graph was not a simple bar chart, because there was a secondary value (average age of values) also displayed on the bars, so it has been adapted to display only a single dependent value.
}

\section{Advertisers Pay More for Youth}

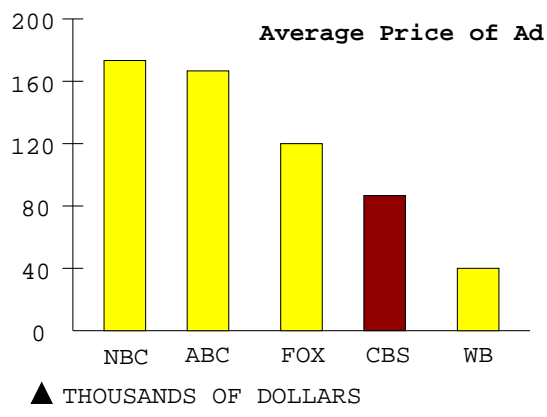

Figure 4: Graphic with a Get-Rank Message ${ }^{4}$

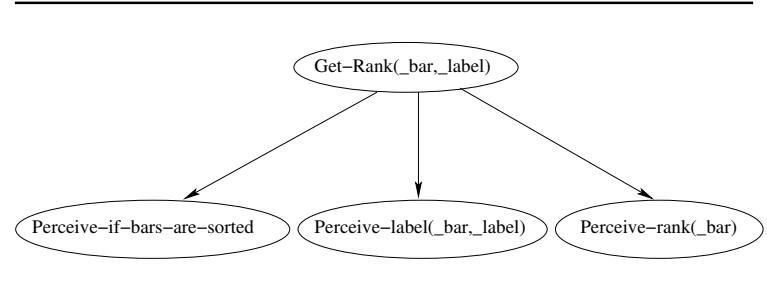

Figure 5: A Piece of Network Structure

Evidence nodes must be added to the Bayesian network to reflect the evidence provided by the information graphic about its intended message. Evidence nodes reflecting the amount of effort required for a perceptual task (categorized as low, medium, high, or impossible) and evidence nodes reflecting whether a parameter of a perceptual task is salient (via highlighting, annotating it, etc.) are attached to perceptual task nodes. For example, consider the graphic in Figure 4, the piece of network structure shown in Figure 5, and the evidence nodes that would be attached to the instantiated perceptual task node Perceiverank(BAR4). The effort evidence node would indicate that little effort is required for this task since the bars are sorted according to height. The highlighting evidence node would indicate that the instantiated parameter BAR4 is highlighted in the graphic. The annotation and noun-in-caption evidence nodes would indicate respectively that no bars have special annotations and that none of the bar labels are part of the graphic's caption. Evidence nodes reflecting the presence of one of our verb or adjective classes in the caption provide evidence for a general category of message and thus are attached to the top level node in the network.

Associated with each child node in a Bayesian network is a conditional probability table that gives the conditional probability for each value of the child node given the value of the parent node. In our 
Bayesian network, the value of the parent node is either that it is or is not part of the plan that the graphic designer has for the viewer to deduce the graphic's message. The conditional probability tables for network nodes are learned from our corpus of graphics.

Once the network with its evidence nodes is built, the probabilities propagate through the network to hypothesize the intended message of the graphic. For the graphic in Figure 4, SIGHT infers that the graphic is conveying the rank of CBS and produces the natural language "This bar chart titled 'Advertisers pay more for youth' shows that CBS has the second lowest rank in terms of the dollar value of average price of Ad compared with NBC, ABC, FOX, and WB." 5 .

\subsubsection{Evaluation}

The performance of the message inference within our SIGHT system has been evaluated in two ways. First, using a corpus of 110 simple bar charts that had previously been annotated with their primary message by two human coders, we evaluated our approach using leave-one-out cross validation in which each graphic is selected once as the test graphic, and the other 109 graphics are used to compute the conditional probability tables for the Bayesian network. We viewed the system as successful in recognizing the graphic's message if its top-rated hypothesis matched the message assigned by the coders and the system-assigned probability for the hypothesis exceeded $50 \%$. The system's overall success rate, $79.1 \%$, is the average of the results of all 110 experiments.

We also performed a qualitative evaluation in order to determine whether the intentions being inferred by our system would meet the approval of users. Seventeen human subjects rated a posited message for each of 27 bar charts. In 20 of the 27 cases, the posited message matched the message inferred by our system. Subjects rated the messages from 0 (strongly disagree) to 4 (strongly agree). For the 20 messages matching the system's output, the rating was 3.33 with a standard deviation of 1.02 and a 95\% confidence interval of .108 , whereas for the 7 messages that differed from the system's output, the rating was only 1.19 with a standard deviation of 1.46 and a $95 \%$ confidence interval of .261 .

From these evaluations, we conclude that our system has a high degree of success at recognizing the primary message of a simple bar chart, and that using the recognized message as the basis for a graphic's summary should produce summaries that would be satisfactory to a majority of users.

\footnotetext{
${ }^{5}$ For further detail on the Bayesian network, see (Elzer et al., 2005b)
}

\subsection{Generating the Summary}

Once the intended message has been inferred by our Bayesian inference system, it is used as the core content of a textual summary of the graphic. One of the most challenging aspects of generating coherent natural language has been determining the full label for the measurement (or value) axis. In examining our corpus of bar charts, taken from a variety of magazines and newspapers, we have found that the measurement axis label might be very abbreviated and that full rendering of the label often requires extraction of words from text within the graphic (such as AVERAGE LAPTOP PRICES in Figure 3) or from the caption and/or second-tier descriptive text below the caption. We have constructed a set of heuristics for ranking the graphic's components in terms of where to look for the measurement axis label and how to extract it from these textual pieces. Other heuristics augment the wording of the label. For example, one heuristic states that if the graphic's text contains a single proper noun that does not match the label of a bar, then the measurement axis label should generally be preceded with that proper noun in possessive case. Consider, for example, the graphic in Figure 6. Here the measurement axis is labelled as Percentage of unauthorized workers and the unit of measurement is also captured by the \% sign after the annotated values, but "Percentage of unauthorized workers" must be preceded with the proper noun "United States" in order to fully describe what is being measured. The natural language generation component of our system (to be presented in a separate paper) uses a set of heuristics to generate a complete rendering of the measurement axis label, which is then used in templates to generate the appropriate wording for the particular category of inferred message. For example, SIGHT hypothesizes that the graphic in Figure 6 is conveying a Rank-of-all message and generates the textual summary "This bar chart titled 'Workers without papers' compares the entities Farming, Cleaning, Construction, and Food preparation with respect to United States's percentage of unauthorized workers."

\section{CONCLUSION}

SIGHT has been implemented and tested for simple bar charts. We eventually envision SIGHT as an interactive natural language system which infers the intended message of an information graphic, provides a summary that includes the intended message along with notable features of the graphic, and then responds to follow-up questions from the user. These 


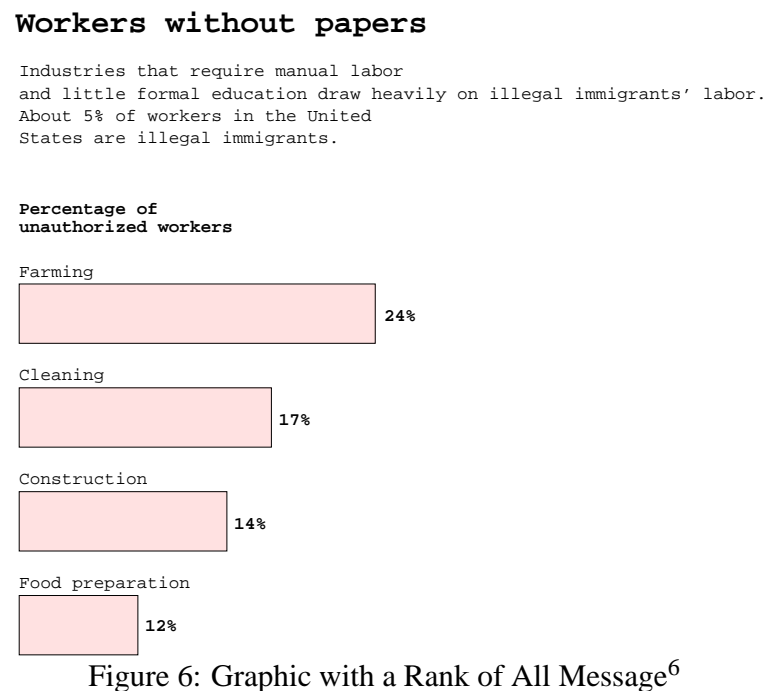

additional features would obviously make for a richer and more robust user interface. We are also working to make the image processing in VEM more robust, and to extend the SIGHT system to other kinds of information graphics such as line graphs and pie charts, and to complex graphics, such as grouped bar charts.

Information graphics are an important part of many documents available on the world-wide web, yet they are largely inaccessible to visually impaired users. This paper has presented a novel implemented interface that enables visually impaired users to gain access to the information provided by simple bar charts that appear on a web page. Our approach of presenting the message conveyed by the information graphic, rather than rendering the graphic in a different medium, has significant advantages — it provides the user with easy access to the communicative intent of the graphic, and does not require specialized hardware or for the user to construct a mental map of the graphic. Moreover, our system does not require any action on the part of the web page developer.

\section{REFERENCES}

Alty, J. L. and Rigas, D. (1998). Communicating graphical information to blind users using music: The role of context. In Proc. of CHI-98, Human Factors in Computer Systems, p. 574-581, Los Angeles. ACM Press.

Carberry, S., Elzer, S., and Demir, S. (2006). Information graphics: An untapped resource for digital libraries. In Proc. of SIGIR 2006, Seattle, WA.

\footnotetext{
${ }^{6}$ Graphic from USA Today, July 11, 2006 issue.
}

Chester, D. and Elzer, S. (2005). Getting computers to see information graphics so users do not have to. In Proc. of the 15th Int'l Symposium on Methodologies for Intelligent Systems, LNAI 3488, p. 660-668. Springer-Verlag.

Elzer, S., Carberry, S., Chester, D., Demir, S., Green, N., Zukerman, I., and Trnka, K. (2005a). Exploring and exploiting the limited utility of captions in recognizing intention in information graphics. In Proc. of the 43rd Annual Meeting of the ACL, p. 223-230.

Elzer, S., Carberry, S., Zukerman, I., Chester, D., Green, N., and Demir, S. (2005b). A probabilistic framework for recognizing intention in information graphics. In Proc. of IJCAI, p. 10421047.

Elzer, S., Green, N., Carberry, S., and Hoffman, J. (2006). A model of perceptual task effort for bar charts and its role in recognizing intention. User Modeling and User-Adapted Interaction, 16(1):1-30.

Ina, S. (1996). Computer graphics for the blind. ACM SIGCAPH Computers and the Physically Handicapped, 55:16-23.

Kennel, A. R. (1996). Audiograf: A diagram-reader for the blind. In Second Annual ACM Conference on Assistive Technologies, p. 51-56.

Kerpedjiev, S. and Roth, S. (2000). Mapping communicative goals into conceptual tasks to generate graphics in discourse. In Proc. of the Int'l Conf. on Intelligent User Interfaces, p. 60-67.

Kurze, M. (1995). Giving blind people access to graphics (example: Business graphics). In Proc. Software-Ergonomie '95 Workshop Nicht-visuelle graphische Benutzungsoberflchen, Darmstadt, Germany.

Larkin, J. and Simon, H. (1987). Why a diagram is (sometimes) worth ten thousand words. Cognitive Science, 11:65-99.

Meijer, P. B. (1992). An experimental system for auditory image representations. IEEE Transactions on Biomedical Engineering, 39(2):112-121.

Ramloll, R., Yu, W., Brewster, S., Riedel, B., Murton, M., and Dimigen, G. (2000). Constructing sonified haptic line graphs for the blind student: First steps. In Proc. of ASSETS 2000, p. 17-25, Arlington, VA.

Yu, W., Reid, D., and Brewster, S. (2002). Web-based multimodal graphs for visually impaired people. In Proc. of the 1st Cambridge Workshop on Universal Access and Assistive Techn., p. 97-108. 